\title{
A Bayesian framework with auxiliary particle filter for GMTI based ground vehicle tracking aided by domain knowledge
}

\author{
Miao Yu ${ }^{a}$, Cunjia Liu ${ }^{a}$, Wen-hua Chen $^{a}$ and Jonathon Chambers ${ }^{b}$ \\ ${ }^{a}$ Department of Aeronautical and Automotive Engineering, Loughborough \\ University, UK, LE11 3TU (e-mail: \{m.yu, c.liu5, w.chen\}@lboro.ac.uk); \\ ${ }^{b}$ School of Electronic, Electrical and Systems Engineering, Loughborough \\ University, UK, LE11 3TU (e-mail: J.A.Chambers@lboro.ac.uk)
}

\begin{abstract}
In this work, we propose a new ground moving target indicator (GMTI) radar based ground vehicle tracking method which exploits domain knowledge. Multiple state models are considered and a Monte-Carlo sampling based algorithm is preferred due to the manoeuvring of the ground vehicle and the non-linearity of the GMTI measurement model. Unlike the commonly used algorithms such as the interacting multiple model particle filter (IMMPF) and bootstrap multiple model particle filter (BS-MMPF), we propose a new algorithm integrating the more efficient auxiliary particle filter (APF) into a Bayesian framework. Moreover, since the movement of the ground vehicle is likely to be constrained by the road, this information is taken as the domain knowledge and applied together with the tracking algorithm for improving the tracking performance. Simulations are presented to show the advantages of both the new algorithm and incorporation of the road information by evaluating the root mean square error (RMSE).
\end{abstract}

\section{INTRODUCTION}

This paper focuses on the problem of tracking a ground vehicle moving on the road by a GMTI radar sensor. A ground vehicle could manoeuvre with different movement types (such as acceleration, constant velocity and stop) constrained by the road. Measurements from a GMTI radar are used to discriminate a moving target from the stationary background, such as the range and azimuth angle of the tracked object. As mentioned in [1], a GMTI radar sensor is capable of detecting targets moving on the ground for wide-area, day/night and all weather conditions. Successful tracking of single/multiple ground vehicle(s) moving on a road is important for traffic surveillance.

The aim of vehicle tracking is to estimate the vehicle's state values (including positions and velocities) according to the measurements provided by the sensor. Various algorithms have been applied to solve the tracking problem, the standard ones include the Kalman Filter and its variations, and the particle filter [2].

\footnotetext{
${ }^{*}$ This work was supported by the Engineering and Physical Sciences Research Council (EPSRC) Grant number EP/K014307/1 and the MOD University Defence Research Collaboration in Signal Processing.
} 
As the tracked ground vehicle could manoeuvre with different movement types, multiple state models are needed to describe the movement of the tracked vehicle and then a stochastic hybrid system (SHS) can be constructed, which has different modes (corresponding to different state models) governed by a discrete stochastic process. Each mode is from a set of a finite number of possible modes and the transition between one mode to another, between consecutive time instances, follows a certain probability.

Standard Kalman/particle filters can only deal with one state model so that they are not applicable for solving the SHS problem in which multiple state models are involved. In order to obtain the state estimation in an SHS, E. Mazor et al. [3] proposed an interactive multiple model (IMM) method. The KF/EKFs are applied in the IMM to obtain the state estimates for every state model, which are combined to obtain the state estimation result. The initial conditions for the KF/EKFs corresponding to a particular state model are obtained as a mixture of the estimation results for all the state models at the previous time instance. Considering the fact that the state distribution for every mode of an SHS could not be a single Gaussian and the measurement model may be non-linear (such as the GMTI based measurement model), instead of Kalman filters, Y. Boers et al. in [4] implemented the particle filtering in the IMM framework to obtain an IMMPF algorithm for solving the non-linear and non-Gaussian problems. H. Blom et al. in [5] extended the IMMPF in [4] from a Markov-jump system to a non-Markov system, considering the mode transition probabilities are not constant but depend on state vectors in some realistic scenarios.

Except for the algorithm side, we also need to highlight that information such as the road boundary, directions, speed limits and so forth, can be fused to reduce the uncertainty of the target motion to obtain a more accurate state estimation result. In [6], the vehicle state is augmented with a mode variable which indicates whether the vehicle is on the road or not, and a sampling importance resampling (SIR) particle filter is applied to estimate the augmented state. A separate model is applied for describing the vehicle's on-road movement; in this way the road information is exploited. M. Ulmke et al. in [7] made use of the road map information by considering a more complicated road topography; besides, the road map errors were also taken into account. Two schemes, including a framework of Gaussian sum approximations and a particle filtering approach, were applied to incorporate the road information for state estimation. A hybrid method is applied in [8], in which the vehicle on the road moves in multiple modes. The target state space is regarded as a hybrid state space which is partitioned into the mode subspace and the target subspace which contains other state values (such as the target's position and velocity). And the hybrid state is estimated by a more efficient Rao-Blackwellization particle filtering scheme.

For a realistic scenario, the changes of the vehicle's movement modes are related to the vehicle's state components, such as positions and velocities. For example, the vehicle is more likely to decelerate when it approaches a junction and the vehicle is less likely to stop suddenly when it moves at a high velocity. So, a non-Markov jump system in [5] which adopts statedependent mode transition probability (SDTP) should be applied to reflect the mode transition of the vehicle's movement. Besides, it is more realistic to consider the width of the road instead of simply modeling the road segmentation as 1-D straight line ([6], [7] and [8]) with the width being zero, which conflicts with the realistic scenario.

In this work, we propose a novel GMTI radar based ground vehicle tracking method. The recursive Bayesian framework in [5] is adopted for a non-Markov jump system. But instead 
of a generic particle filter to implement the Bayesian framework, an auxiliary particle filtering (APF) scheme is applied to make use of the measurement information to obtain a more accurate result. Moreover, we exploit the road information as the domain knowledge to further improve the tracking results.

This paper is organized as follows: Section 2 proposes the general framework of the road information aided ground vehicle tracking problem by applying a GMTI radar. The recursive Bayesian framework for a non-Markov jump system is proposed in Section 3. In Section 4, the implementation of the recursive Bayesian framework by the auxiliary particle filter is explained in detail. Section 5 finally shows the simulation results for GMTI tracking of a vehicle moving on a road, which confirms the advantages of our proposed method.

\section{GENERAL PROBLEM FORMULATION}

\subsection{Road information aided multiple model estimation}

We assume that the ground vehicle can move in different modes (acceleration, constant velocity and stop) and its movement is constrained by the road. So, the state model used to describe the movement of the ground vehicle could be regarded as an SHS with constraints. Generally, the vehicle dynamics on the road can be represented as:

$$
\mathbf{x}_{t}=f\left(\mathbf{x}_{t-1}, m_{t}, \mathbf{C}_{t}, \mathbf{w}_{t-1}\right)
$$

where $\mathbf{x}_{t} \in \mathbf{R}^{n}$ denotes the target state (including the positions and velocities of the tracked vehicle) at time instant $t, m_{t}$ is the discrete mode state used to indicate the movement mode of the vehicle, $\mathbf{w}_{t}$ is the process noise and $\mathbf{C}_{t}$ represents the constraints of the target state $\mathbf{x}_{t}$.

Road information directly determines the constraint region $\mathbf{C}_{t}$. For example, the road boundary and speed limits restrict the target's position and velocity to be within certain intervals. By imposing the target's state vector $\mathbf{x}_{t}$ in the region $\mathbf{C}_{t}$ instead of the whole $\mathbf{R}^{n}$ space, the uncertainty of $\mathbf{x}_{t}$ is thereby reduced.

Additionally, other road information is related to the movement mode transitions. One example is that the vehicle is more likely to decelerate when it approaches a bend in the road. This type of information could be incorporated into the mode transition probabilities by making them dependent on the state vector. For instance, high transition probability to 'decelerate' mode is assigned when the vehicle's position is near the road turn. By adopting the statedependent mode transition probability (SDTP), the SHS then becomes a non-Markov jump system.

\subsection{Vehicle movement model}

For describing the vehicle's movement, two types of coordinate systems are considered. The first is a global Cartesian coordinate system $O X Y$. The second coordinate system is a local curvilinear coordinate system associated with the road. The origin of the local curvilinear coordinate system is assigned to the starting point of the road denoted as $e_{r}$. The first axis aligns with the continuous curve representing the center line of the road, whereas the second axis is along the normal direction of the curve. In the local curvilinear coordinate system, the vehicle position can be expressed by using the travelled distance $l_{r}$ along the center line from starting junction and the deviation $d_{r}$ from the center line of the current road. Examples 
of the global Cartesian coordinate system and local curvilinear coordinate system are shown in Figure 1. These two coordinates could be converted to each other by applying the corresponding transformation operations $T_{g}^{r}(\cdot)$ and $T_{r}^{g}(\cdot)$ (which represent the transformation for the global coordinate to local road coordinate and local road coordinate to the global one respectively, and the detailed definitions of these functions could be found in [9]).

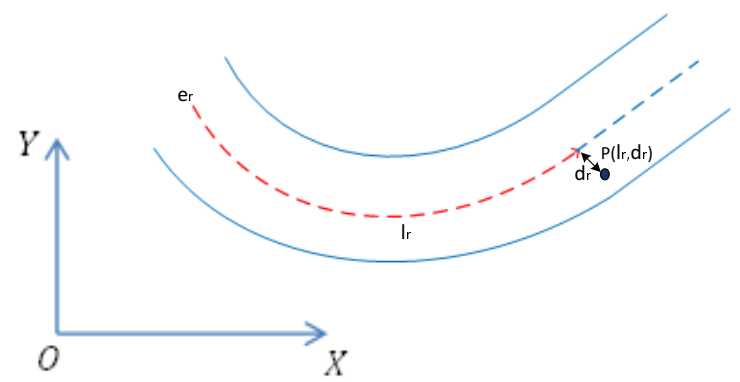

Figure 1. The illustration of two types of coordinate systems. The position of a point $P$ (representing a vehicle) on the road could be determined by the travelled distance along the centerline $l_{r}$ (marked as red) and the normal distance to the centerline $d_{r}$.

In this work, the vehicle movement is modeled in the local curvilinear coordinate, in which the state of the target on the road $r$ at time $t$ is defined as $\mathbf{x}_{t}^{r}=\left[\begin{array}{llll}l_{t} & i_{t} & d_{t} & \dot{d}_{t}\end{array}\right]^{T}$, where $(\cdot)^{T}$ denotes vector transpose. Here $l_{t}$ and $d_{t}$ represent the positions along and perpendicular to the centerline, and $\dot{l}_{t}$ and $\dot{d}_{t}$ represent the corresponding velocities. One advantage of using the local curvilinear coordinate is the implicit integration of curvature information in the model without considering a turning motion. In addition, the road width constraint can be easily incorporated in to the model as $a \leq d_{t} \leq b$, where $[a, b]$ represents the feasible region for $d_{t}$, which is the distance perpendicular to the centerline.

And a normally chosen state transition model is a constant velocity (CV) model [2] with the following definition:

$$
\mathbf{x}_{t}^{r}=F \cdot \mathbf{x}_{t-1}^{r}+G \cdot \mathbf{w}_{t}^{r}\left(m_{t}\right)
$$

where

$$
F=\left[\begin{array}{cccc}
1 & T & 0 & 0 \\
0 & 1 & 0 & 0 \\
0 & 0 & 1 & T \\
0 & 0 & 0 & 1
\end{array}\right], \quad G=\left[\begin{array}{cc}
T^{2} / 2 & 0 \\
T & 0 \\
0 & T^{2} / 2 \\
0 & T
\end{array}\right]
$$

and $T$ is the sampling time, $\mathbf{w}_{t}^{r}\left(m_{t}\right)$ is the Gaussian process noise representing the acceleration components controlled by the movement mode $m_{t}$, which has zero means and covariance matrix $\Sigma\left(m_{t}\right)=\operatorname{diag}\left\{\sigma_{l}^{r}\left(m_{t}\right), \sigma_{d}^{r}\left(m_{t}\right)\right\}$.

\subsection{Measurement model}

As mentioned in [10], the standard GMTI radar could measure the range and azimuth angle (denoted as $r_{t}$ and $\theta_{t}$ at time instance $t$ ) of the ground vehicle relative to the position of the 
GMTI radar. By assuming these measurements are noise-corrupted from the actual values, we have:

$$
\mathbf{y}_{t}=\left[\begin{array}{c}
r_{t} \\
\theta_{t}
\end{array}\right]=\left[\begin{array}{c}
\sqrt{\left(x_{o}-x_{t}\right)^{2}+\left(y_{o}-y_{t}\right)^{2}+\left(z_{o}\right)^{2}} \\
\arctan \left(\frac{y_{o}-y_{t}}{x_{o}-x_{t}}\right)
\end{array}\right]+\mathbf{n}_{t}
$$

for a received measurement $\mathbf{y}_{t}$. Here $\left(x_{t}, y_{t}\right)$ represent the vehicle's position in the global coordinate system at time $t$, which is converted from the local road coordinate $\mathbf{x}_{t}^{r}$ by $T_{r}^{g}(\cdot)$ (assuming the ground plane's z-coordinate in the global coordinate is zero $),\left(x_{o}, y_{o}, z_{o}\right)$ represents the position of the observer (GMTI radar) and $\mathbf{n}_{t}$ represents the measurement noises. Similar to the process noise, we assume that it also follows a Gaussian distribution with zero means and covariance matrix denoted as $\left[\begin{array}{cc}\sigma_{r}^{2} & 0 \\ 0 & \sigma_{\theta}^{2}\end{array}\right]$.

\section{IMM BAYESIAN ESTIMATION FRAMEWORK}

In this section, we discuss the exact recursive Bayesian derivations for a non-Markov jump system. Given the measurement sequence $Z^{t}=\left\{\mathbf{z}_{1}, \ldots, \mathbf{z}_{t}\right\}$ and the constraint $\mathbf{C}^{t}$, the probability density function $p\left(\mathbf{x}_{t-1}, m_{t-1} \mid \mathbf{Z}^{t-1}, \mathbf{C}^{t-1}\right)$ is updated to $p\left(\mathbf{x}_{t}, m_{t} \mid \mathbf{Z}^{t}, \mathbf{C}^{t}\right)$ at $t$ by four main steps: mode mixing, state intersection, evolution and correction:

$$
\begin{gathered}
p\left(m_{t-1} \mid \mathbf{Z}^{t-1}, \mathbf{C}^{t-1}\right) \stackrel{\text { Mixing }}{\longrightarrow} p\left(m_{t} \mid \mathbf{Z}^{t-1}, \mathbf{C}^{t-1}\right) \\
p\left(\mathbf{x}_{t-1} \mid m_{t-1}, \mathbf{Z}^{t-1}, \mathbf{C}^{t-1}\right) \stackrel{\text { Interact }}{\longrightarrow} p\left(\mathbf{x}_{t-1} \mid m_{t}, \mathbf{Z}^{t-1}, \mathbf{C}^{t-1}\right) \\
p\left(\mathbf{x}_{t-1} \mid m_{t}, \mathbf{Z}^{t-1}, \mathbf{C}^{t-1}\right) \stackrel{\text { Evolutions }}{\longrightarrow} p\left(\mathbf{x}_{t} \mid m_{t}, \mathbf{Z}^{t-1}, \mathbf{C}^{t-1}\right) \\
p\left(\mathbf{x}_{t} \mid m_{t}, \mathbf{Z}^{t-1}, \mathbf{C}^{t-1}\right) \stackrel{\text { Correction }}{\longrightarrow} p\left(\mathbf{x}_{t}, m_{t} \mid \mathbf{Z}^{t}, \mathbf{C}^{t}\right)
\end{gathered}
$$

\subsection{Mode mixing}

The evolution of the conditional mode probability from $t-1$ to $t$ is characterized as the mode mixing transition. Using the law of total probability, it yields

$$
\begin{aligned}
& p\left(m_{t} \mid Z^{t-1}, \mathbf{C}^{t-1}\right)=\sum_{m_{t-1} \in \mathcal{M}} p\left(m_{t}, m_{t-1} \mid Z^{t-1}, \mathbf{C}^{t-1}\right) \\
& =\sum_{m_{t-1} \in \mathcal{M}} p\left(m_{t} \mid m_{t-1}, Z^{t-1}, \mathbf{C}^{t-1}\right) p\left(m_{t-1} \mid Z^{t-1}, \mathbf{C}^{t-1}\right)
\end{aligned}
$$

where $\mathcal{M}$ represents the collection of the mode indices and $p\left(m_{t} \mid m_{t-1}, Z^{t-1}, \mathbf{C}^{t-1}\right)$ can be obtained using the law of total probability as:

$$
\begin{aligned}
& p\left(m_{t} \mid m_{t-1}, Z^{t-1}, \mathbf{C}^{t-1}\right) \\
& =\int p\left(m_{t} \mid m_{t-1}, \mathbf{x}_{t-1}\right) \cdot p\left(\mathbf{x}_{t-1} \mid m_{t-1}, \mathbf{Z}^{t-1}, \mathbf{C}^{t-1}\right) \mathrm{d} \mathbf{x}_{t-1}
\end{aligned}
$$




\subsection{State interaction}

The state interaction process is carried out for each mode, to generate the initial mode-conditioned density $p\left(\mathbf{x}_{t-1} \mid m_{t}, \mathbf{Z}^{t-1}, \mathbf{C}^{t-1}\right)$. Again, by using the laws of conditional/total probability, we can obtain:

$$
\begin{aligned}
& p\left(\mathbf{x}_{t-1} \mid m_{t}, \mathbf{Z}^{t-1}, \mathbf{C}^{t-1}\right)=\frac{p\left(\mathbf{x}_{t-1}, m_{t} \mid \mathbf{Z}^{t-1}, \mathbf{C}^{t-1}\right)}{p\left(m_{t} \mid \mathbf{Z}^{t-1}, \mathbf{C}^{t-1}\right)} \\
& =\frac{\sum_{m_{t-1} \in \mathcal{M}} p\left(m_{t} \mid m_{t-1}, \mathbf{x}_{t-1}\right) \cdot p\left(\mathbf{x}_{t-1}, m_{t-1} \mid \mathbf{Z}^{t-1}, \mathbf{C}^{t-1}\right)}{p\left(m_{t} \mid \mathbf{Z}^{t-1}, \mathbf{C}^{t-1}\right)}
\end{aligned}
$$

\subsection{Evolution}

The state evolution step is to propagate the mode-conditioned state density $p\left(\mathbf{x}_{t-1} \mid m_{t}, \mathbf{Z}^{t-1}, \mathbf{C}^{t-1}\right)$ at $t-1$ to $p\left(\mathbf{x}_{t} \mid m_{t}, \mathbf{Z}^{t-1}, \mathbf{C}^{t}\right)$. Given the initial density provided in (11), the mode-conditioned state density $p\left(\mathbf{x}_{t} \mid m_{t}, Z^{t-1}, \mathbf{C}^{t}\right)$ can be calculated via:

$$
\begin{aligned}
& p\left(\mathbf{x}_{t} \mid m_{t}, \mathbf{Z}^{t-1}, \mathbf{C}^{t}\right) \\
& =\int p\left(\mathbf{x}_{t-1} \mid m_{t}, \mathbf{Z}^{t-1}, \mathbf{C}^{t-1}\right) p\left(\mathbf{x}_{t} \mid \mathbf{x}_{t-1}, m_{t}, \mathbf{Z}^{t-1}, \mathbf{C}^{t}\right) \mathrm{d} \mathbf{x}_{t-1}
\end{aligned}
$$

where $p\left(\mathbf{x}_{t} \mid \mathbf{x}_{t-1}, m_{t}, \mathbf{Z}^{t-1}, \mathbf{C}^{t}\right)$ is the state transition probability determined by both the state model and constraints, which follows:

$$
p\left(\mathbf{x}_{t} \mid \mathbf{x}_{t-1}, m_{t}, \mathbf{Z}^{t-1}, \mathbf{C}^{t}\right) \propto \begin{cases}p\left(\mathbf{x}_{t} \mid \mathbf{x}_{t-1}, m_{t}, \mathbf{Z}^{t-1}\right) & \text { if } \mathbf{x}_{t} \in \mathbf{C}_{t} \\ 0 & \text { otherwise }\end{cases}
$$

when $\mathbf{x}_{t}$ is not within the constraint $\mathbf{C}_{t}$, the probability becomes zero; otherwise, it is calculated by $p\left(\mathbf{x}_{t} \mid \mathbf{x}_{t-1}, m_{t}, \mathbf{Z}^{t-1}\right)$, which is determined by the dynamics of the state model $m_{t}$.

\subsection{Correction}

Finally, the density function $p\left(\mathbf{x}_{t}, m_{t} \mid \mathbf{Z}^{t}, \mathbf{C}^{t}\right)$ conditioned on the current measurements and constraints is obtained. According to the Bayesian formula, we have:

$$
p\left(\mathbf{x}_{t}, m_{t} \mid \mathbf{Z}^{t}, \mathbf{C}^{t}\right)=\frac{p\left(\mathbf{z}_{t} \mid \mathbf{x}_{t}\right) \cdot p\left(\mathbf{x}_{t}, m_{t} \mid \mathbf{Z}^{t-1}, \mathbf{C}^{t}\right)}{p\left(\mathbf{z}_{t} \mid \mathbf{Z}^{t-1}\right)}
$$

By substituting the results of the mode mixing step and evolution step, $p\left(m_{t} \mid \mathbf{Z}^{t-1}\right)$ and $p\left(\mathbf{x}_{t} \mid m_{t}, \mathbf{Z}^{t-1}\right)$, into (15) finally we can obtain:

$$
\begin{aligned}
p\left(\mathbf{x}_{t}, m_{t} \mid \mathbf{Z}^{t}, \mathbf{C}^{t}\right) & =\frac{p\left(\mathbf{z}_{t} \mid \mathbf{x}_{t}\right) \cdot p\left(\mathbf{x}_{t} \mid m_{t}, \mathbf{Z}^{t-1}, \mathbf{C}^{t}\right) \cdot p\left(m_{t} \mid \mathbf{Z}^{t-1}, \mathbf{C}^{t-1}\right)}{p\left(\mathbf{z}_{t} \mid \mathbf{Z}^{t-1}\right)} \\
& \propto p\left(\mathbf{z}_{t} \mid \mathbf{x}_{t}, m_{t}\right) \cdot p\left(\mathbf{x}_{t} \mid m_{t}, \mathbf{Z}^{t-1}, \mathbf{C}^{t}\right) \cdot p\left(m_{t} \mid \mathbf{Z}^{t-1}, \mathbf{C}^{t-1}\right)
\end{aligned}
$$

At this point the IMM Bayesian filtering cycle is finished and the probability density function $p\left(\mathbf{x}_{t-1}, m_{t-1} \mid \mathbf{Z}^{t-1}, \mathbf{C}^{t-1}\right)$ at time instance $t-1$ is propagated to $p\left(\mathbf{x}_{t}, m_{t} \mid \mathbf{Z}^{t}, \mathbf{C}^{t}\right)$ at time $t$. 
The mode probability $p\left(m_{t} \mid \mathbf{Z}^{t}, \mathbf{C}^{t}\right)$ and mode-conditioned probability $p\left(\mathbf{x}_{t} \mid m_{t}, \mathbf{Z}^{t}, \mathbf{C}^{t}\right)$ could be derived as:

$$
\begin{aligned}
& p\left(m_{t} \mid \mathbf{Z}^{t}, \mathbf{C}^{t}\right)=\int_{\mathbf{x}_{t}} p\left(\mathbf{x}_{t}, m_{t} \mid \mathbf{Z}^{t}, \mathbf{C}^{t}\right) \mathrm{d} \mathbf{x}_{t} \\
& p\left(\mathbf{x}_{t} \mid m_{t}, \mathbf{Z}^{t}, \mathbf{C}^{t}\right)=\frac{p\left(\mathbf{x}_{t}, m_{t} \mid \mathbf{Z}^{t-1}, \mathbf{C}^{t}\right)}{p\left(m_{t} \mid \mathbf{Z}^{t}, \mathbf{C}^{t}\right)}
\end{aligned}
$$

where $p\left(m_{t} \mid \mathbf{Z}^{t}, \mathbf{C}^{t}\right)$ represents the likelihood of a particular mode. The mode with the largest likelihood is regarded as the current movement type of the tracked object (denoted as mode $_{t}$ ). The maximum a posterior (MAP) or minimum mean square (MMS) estimators of the state vector (Ristic and Gordon (2004)) for mode $e_{t}$ could be taken as the final state estimation, which could be obtained from the mode-conditioned probability $p\left(\mathbf{x}_{t} \mid \operatorname{mode}_{t}, \mathbf{Z}^{t}, \mathbf{C}^{t}\right)$.

\section{IMM AUXILIARY PARTICLE FILTER IMPLEMENTATION}

As there is not an exact analytical solution for $p\left(\mathbf{x}_{t}, m_{t} \mid \mathbf{Z}^{t}, \mathbf{C}^{t}\right)$ due to the non-Gaussian property of the state distribution for a non-Markov jump SHS and the non-linear property of the measurement model, to obtain a more accurate probability density function estimation, a particle filtering scheme [2] is applied to implement the IMM based Bayesian filtering. However, instead of the generic particle filtering scheme, we apply the auxiliary particle filtering method, which takes the measurements into account to obtain a more accurate estimation. And we denote the IMM based Bayesian filtering implemented by the auxiliary particle filter as IMMAPF.

At time $t-1$, we assume that $N$ weighted particles are allocated to the corresponding mode-matched filter as: $\left\{\mathbf{x}_{t-1}^{r, k}, w_{t-1}^{r, k} ; k=1, \ldots, N\right\}$ for each mode $r \in \mathcal{M}$, which are used to approximate the joint distribution $p\left(\mathbf{x}_{t-1}, m_{t-1}=r \mid Z^{t-1}\right)$ as:

$$
p\left(\mathbf{x}_{t-1}, m_{t-1}=r \mid \mathbf{Z}^{t-1}, \mathbf{C}^{t-1}\right) \approx \sum_{k=1}^{N} w_{t-1}^{r, k} \delta\left(\mathbf{x}_{t-1}-\mathbf{x}_{t-1}^{r, k}\right)
$$

where $\delta(\cdot)$ is a Dirac delta function.

The approximation in (17), could be applied to implement the recursive IMM Bayesian derivation framework. For the mode mixing step, the prior mode probability for mode $m_{t}=s$ can be approximated as:

$$
\begin{aligned}
p\left(m_{t}=s \mid Z^{t-1}, \mathbf{C}^{t-1}\right) & \approx \sum_{r \in \mathcal{M}} \sum_{k=1}^{N} p\left(m_{t}=s \mid m_{t-1}=r, \mathbf{x}_{t-1}^{r, k}\right) \cdot w_{t-1}^{r, k} \\
& \triangleq \Lambda_{t-1}^{s}
\end{aligned}
$$

where the notation $\Lambda_{t-1}^{s}$ is defined to facilitate the remainder of the derivation. 
The initial condition probability for mode $m_{t}=s$ could be obtained by substituting (17) and (18) into (11), such that

$$
\begin{aligned}
& p\left(\mathbf{x}_{t-1} \mid m_{t}=s, \mathbf{Z}^{t-1}, \mathbf{C}^{t-1}\right) \\
& \approx \sum_{r \in \mathcal{M}} \sum_{k=1}^{N} p\left(m_{t}=s \mid m_{t-1}=r, \mathbf{x}_{t-1}^{r, k}\right) w_{t-1}^{r, k} \delta\left(\mathbf{x}_{t-1}-\mathbf{x}_{t-1}^{r, k}\right) / \Lambda_{t-1}^{s}
\end{aligned}
$$

Assuming the number of the state modes to be $M$, from equation (19) we can see that $N \times M$ particles are required to represent $p\left(\mathbf{x}_{t-1} \mid m_{t}=s, \mathbf{Z}^{t-1}, \mathbf{C}^{t-1}\right)$, which is the initial density for the $s$-th mode-matched filter. Increasing the number of particles from $N$ to $N \times M$ for probability density function representation will make the number of particles grow in an exponential way as the time increases $((5))$. In order to solve this problem, a resampling step is performed on (19) such that $N$ samples $\left\{\overline{\mathbf{x}}_{t-1}^{s, k}, \bar{w}_{t-1}^{s, k}\right\} \sim p\left(\mathbf{x}_{t-1} \mid m_{t}=s, \mathbf{Z}^{t-1}, \mathbf{C}^{t-1}\right)$ are generated for each mode to represent $p\left(\mathbf{x}_{t-1} \mid m_{t}=s, \mathbf{Z}^{t-1}, \mathbf{C}^{t-1}\right)$, which can be represented as:

$$
p\left(\mathbf{x}_{t-1} \mid m_{t}=s, \mathbf{Z}^{t-1}, \mathbf{C}^{t-1}\right) \approx \sum_{k=1}^{N} \bar{w}_{t-1}^{s, k} \delta\left(\mathbf{x}_{t-1}-\overline{\mathbf{x}}_{t-1}^{s, k}\right)
$$

According to the obtained samples $\left\{\overline{\mathbf{x}}_{t-1}^{s, k}, \bar{w}_{t-1}^{s, k}\right\}_{k=1, \ldots, N}$ for a particular mode $s$, the mode probability $p\left(m_{t}=s \mid \mathbf{Z}^{t}, \mathbf{C}^{t}\right)$ and the mode-conditioned probability $p\left(\mathbf{x}_{t} \mid m_{t}=s, \mathbf{Z}^{t}, \mathbf{C}^{t}\right)$ could be calculated.

\subsection{Calculating mode probability $p\left(m_{t}=s \mid \mathbf{Z}^{t}, \mathbf{C}^{t}\right)$}

The mode probability $p\left(m_{t}=s \mid \mathbf{Z}^{t}, \mathbf{C}^{t}\right)$ could be obtained similar to the derivations in [5]. For each particle $\overline{\mathbf{x}}_{t-1}^{s, k}$, a new particle $\mathbf{x}_{t}^{s, k}$ can be generated according to the state proposal distribution as defined in equation (13). The generated particles $\left\{\mathbf{x}_{t}^{s, k}\right\}_{k=1, \ldots, N}$ are then applied to approximate $p\left(\mathbf{x}_{t} \mid m_{t}=s, \mathbf{Z}^{t-1}, \mathbf{C}_{t}\right)$ according to (12):

$$
p\left(\mathbf{x}_{t} \mid m_{t}=s, \mathbf{Z}^{t-1}, \mathbf{C}_{t}\right) \approx \sum_{k=1}^{N} \bar{w}_{t-1}^{s, k} \delta\left(\mathbf{x}_{t}-\mathbf{x}_{t}^{s, k}\right)
$$

The obtained $p\left(\mathbf{x}_{t} \mid m_{t}=s, \mathbf{Z}^{t-1}, \mathbf{C}_{t}\right)$ together with $\Lambda_{t-1}^{s}$ in (18) could be inserted into (15) which approximates the joint distribution $p\left(\mathbf{x}_{t}, m_{t}=s \mid \mathbf{Z}^{t}, \mathbf{C}_{t}\right)$ as:

$$
\begin{aligned}
p\left(\mathbf{x}_{t}, m_{t}=s \mid \mathbf{Z}^{t}, \mathbf{C}_{t}\right) & \propto \sum_{k=1}^{N} p\left(\mathbf{z}_{t} \mid \mathbf{x}_{t}^{s, k}\right) \bar{w}_{t-1}^{s, k} \delta\left(\mathbf{x}_{t}-\mathbf{x}_{t}^{s, k}\right) \Lambda_{t-1}^{s} \\
& =w_{t}^{s, k} \delta\left(\mathbf{x}_{t}-\mathbf{x}_{t}^{s, k}\right)
\end{aligned}
$$

where $w_{t}^{s, k} \propto \bar{w}_{t-1}^{s, k} p\left(\mathbf{z}_{t} \mid \mathbf{x}_{t}^{s, k}\right) \Lambda_{t-1}^{s}$ and normalized to satisfy $\sum_{s \in \mathcal{M}} \sum_{k=1}^{N} w_{t}^{s, k}=1$.

The mode probability $p\left(m_{t}=s \mid \mathbf{Z}^{t}\right)$ could be obtained by marginalizing $p\left(\mathbf{x}_{t}, m_{t}=s \mid \mathbf{Z}^{t}, \mathbf{C}_{t}\right)$ with respect to $\mathbf{x}_{t}$, which is approximated by summing the weights with respect to mode $s$ as: 


$$
p\left(m_{t}=s \mid \mathbf{Z}^{t}, \mathbf{C}_{t}\right)=\sum_{k=1}^{N} w_{t}^{s, k}
$$

\subsection{Calculating mode conditioned probability $p\left(\mathbf{x}_{t} \mid m_{t}=s, \mathbf{Z}^{t}, \mathbf{C}^{t}\right)$}

For $m_{t}=s$, an auxiliary particle filtering technique could be applied to estimate the corresponding mode conditioned probability at time instance $t$. Initially, some characterization $\mathbf{u}_{t}^{s, k}$ (such as the mean or a sample) is generated from the state propagation density function for every $\overline{\mathbf{x}}_{t-1}^{s, k}$. The likelihood for the new sample, denoted as $w^{\prime s, k}$ is calculated as $w^{\prime s, k} \propto p\left(\mathbf{z}_{t} \mid \mathbf{u}_{t}^{s, k}\right)$. We resample the obtained sample set $\left\{\overline{\mathbf{x}}_{t-1}^{s, k}\right\}_{k=1, \ldots, N}$ according to obtained weights $\left\{w^{\prime s, k}\right\}_{k=1, \ldots, N}$. And the resampled indices are kept as $\left\{i^{k}\right\}_{k=1, \ldots, N}$.

Prediction is performed according to the obtained resampled indices. For every $k \in\{1, \ldots, N\}$, a new sample $\mathbf{x}_{t}^{s, k}$ could be generated from the state proposal distribution $p\left(\mathbf{x}_{t} \mid \mathbf{x}_{t-1}^{s, i^{k}}, m_{t}=\right.$ $s, \mathbf{Z}^{t-1}, \mathbf{C}^{t}$ ) where $\mathbf{x}_{t-1}^{s, i^{k}}$ is the $i^{k}$ particle for the sample set $\left\{\overline{\mathbf{x}}_{t-1}^{s, k}\right\}_{k=1, \ldots, N}$.

Finally, the weight of every predicted particle $\mathbf{x}_{t}^{s, k}$ is updated as:

$$
\begin{aligned}
w_{t}^{s, k} & \propto \bar{w}_{t-1}^{s, i^{k}} \frac{p\left(\mathbf{z}_{t} \mid \mathbf{x}_{t}^{s, k}\right) p\left(\mathbf{x}_{t}^{s, k} \mid \mathbf{x}_{t-1}^{s, i^{k}}, m_{t}=s, \mathbf{Z}^{t-1}, \mathbf{C}^{t}\right)}{q\left(\mathbf{x}_{t}^{s, k}, i^{k} \mid \mathbf{Z}_{t}, \mathbf{C}^{t}\right)} \\
& =\frac{p\left(\mathbf{z}_{t} \mid \mathbf{x}_{t}^{s, k}\right)}{p\left(\mathbf{z}_{t} \mid \mathbf{u}_{t}^{s, i^{k}}\right)}
\end{aligned}
$$

As mentioned in [2], $q\left(\mathbf{x}_{t}^{s, k}, i^{k} \mid \mathbf{Z}_{t}, \mathbf{C}^{t}\right)$ represents the importance density function with the form:

$$
q\left(\mathbf{x}_{t}^{s, k}, i^{k} \mid \mathbf{Z}_{t}\right) \propto \bar{w}_{t-1}^{s, i^{k}} p\left(\mathbf{z}_{t} \mid \mathbf{u}_{t}^{s, i^{k}}\right) p\left(\mathbf{x}_{t}^{s, k} \mid \mathbf{x}_{t-1}^{s, i^{k}}, m_{t}=s, \mathbf{Z}^{t-1}, \mathbf{C}^{t}\right)
$$

The resulting weights $\left\{w_{t}^{s, k}\right\}_{k=1, \ldots, N}$ are normalized such that $\sum_{k=1}^{N} w_{t}^{s, k}=1$. And the mode conditioned probability could be approximated by the new weights and particles as:

$$
p\left(\mathbf{x}_{t} \mid m_{t}=s, \mathbf{Z}^{t}, \mathbf{C}^{t}\right)=\sum_{k=1}^{N} w_{t}^{s, k} \delta\left(\mathbf{x}_{t}-\overline{\mathbf{x}}_{t}^{s, k}\right)
$$

For all $m_{t} \in \mathcal{M}, p\left(m_{t} \mid \mathbf{Z}^{t}, \mathbf{C}^{t}\right)$ and $p\left(\mathbf{x}_{t} \mid m_{t}, \mathbf{Z}^{t}, \mathbf{C}^{t}\right)$ are calculated. As discussed at the end of Section 3 , the mode with the maximum mode probability value is taken as the current movement mode at time $t$, denoted as mode $_{t}$. The MAP/MMSE estimations of the state vector can be obtained according to $p\left(\mathbf{x}_{t} \mid\right.$ mode $\left._{t}, \mathbf{Z}^{t}\right)$, which is approximated by the weighted particles. 


\section{SIMULATION}

A vehicle is simulated to move on a section of road, which has a width of $10 \mathrm{~m}$. Initially, the vehicle moves at $70 \mathrm{~m} / \mathrm{s}$ for 5 seconds. When the vehicle is near the turn, it decelerates its velocity with a deceleration of $-4 \mathrm{~m} / \mathrm{s}^{2}$ for 6 seconds. The vehicle then increases its velocity to $70 \mathrm{~m} / \mathrm{s}$ again after it passes the turn, with an acceleration of $4 \mathrm{~m} / \mathrm{s}^{2}$. After the acceleration, the vehicle retains the velocity of $70 \mathrm{~m} / \mathrm{s}$ for 6 seconds. A UAV is simulated to fly at a height of $100 \mathrm{~m}$, moving with a circular trajectory with a radius of $100 \mathrm{~m}$ and an angular speed of $\pi / 10$ radians per second for cruising. An onboard GMTI radar is assumed to be applied to measure the relative range and azimuth angle of the tracked vehicle. The simulated scenario is presented in Figure 2.

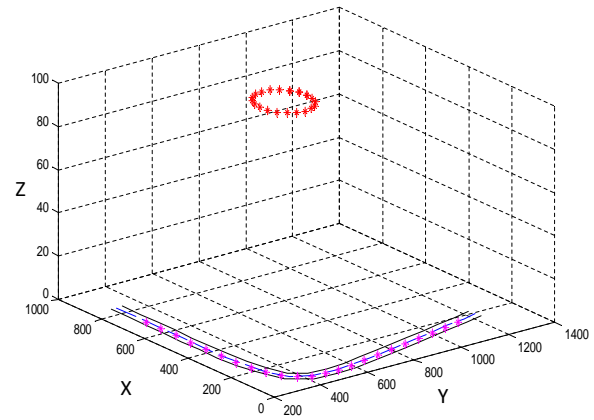

Figure 2. The trajectories of the simulated vehicle and UAV movements. The red star represents the trajectory of the UAV and the pink star represents the trajectory of the vehicle.

Two state models corresponding to the two movement types of constant velocity and acceleration are applied. The state evolutions of the two state models follow equation (2). The process noise $\mathbf{w}$ in these two models follows a Gaussian distribution with zero means. For the acceleration model, the covariance matrix parameters $\sigma_{l}^{r}, \sigma_{d}^{r}$ are set to $\sigma_{l}^{r}=6$ and $\sigma_{d}^{r}=2$. For the constant velocity model, a smaller value of $\sigma_{l}^{r}$ is assigned with $\sigma_{l}^{r}=1$. As mentioned previously, the system is a non-Markov jump system and transition probabilities between different state models are not constant. When the vehicle's position is outside the turn region (marked in the red rectangle in Figure 5), the transition probabilities to two movement modes are set to be equal to introduce no bias towards any movement type as:

$$
\begin{array}{ll}
p\left(m_{t}=C V \mid m_{t-1}=A C\right)=0.5 & p\left(m_{t}=A C \mid m_{t-1}=A C\right)=0.5 \\
p\left(m_{t}=A C \mid m_{t-1}=C V\right)=0.5 & p\left(m_{t}=C V \mid m_{t-1}=C V\right)=0.5
\end{array}
$$

where $C V$ represents constant velocity and $A C$ represents the acceleration.

When the vehicle is in the turn region, we assume the movement mode could only be acceleration and the transition probabilities are:

$$
\begin{array}{ll}
p\left(m_{t}=C V \mid m_{t-1}=A C\right)=0 & p\left(m_{t}=A C \mid m_{t-1}=A C\right)=1 \\
p\left(m_{t}=A C \mid m_{t-1}=C V\right)=1 & p\left(m_{t}=C V \mid m_{t-1}=C V\right)=0
\end{array}
$$




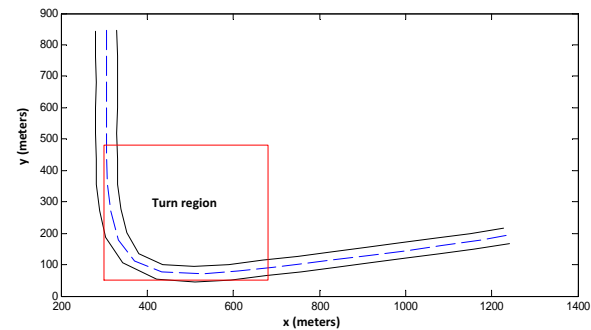

Figure 3. The turn region in which the vehicle accelerates/deccelerates. The $\mathrm{x}$-coordinate range of the turn region is set to $[300,680]$ and the $y$-coordinate range of the turn region is set to $[50,480]$.

The parameters $\sigma_{r}$ and $\sigma_{\theta}$ in the measurement model defined in (4) are set to $\pi / 500$ and 2 .

Three different algorithms, including the bootstrap multiple models particle filter (BSMMPF) [6], IMMPF ([5] and [11]) and our proposed auxiliary particle filter aided IMMPF (IMMAPF) are compared. The first comparison is made without any road information. The comparison results are presented as Figure 4, from which we can see that better performance is obtained for the IMMAPF method with lower root-mean-square-error (RMSE).

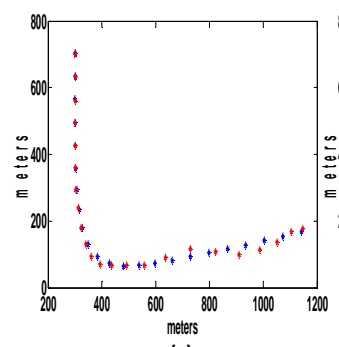

(a)

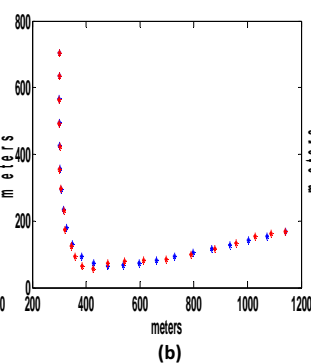

(b)
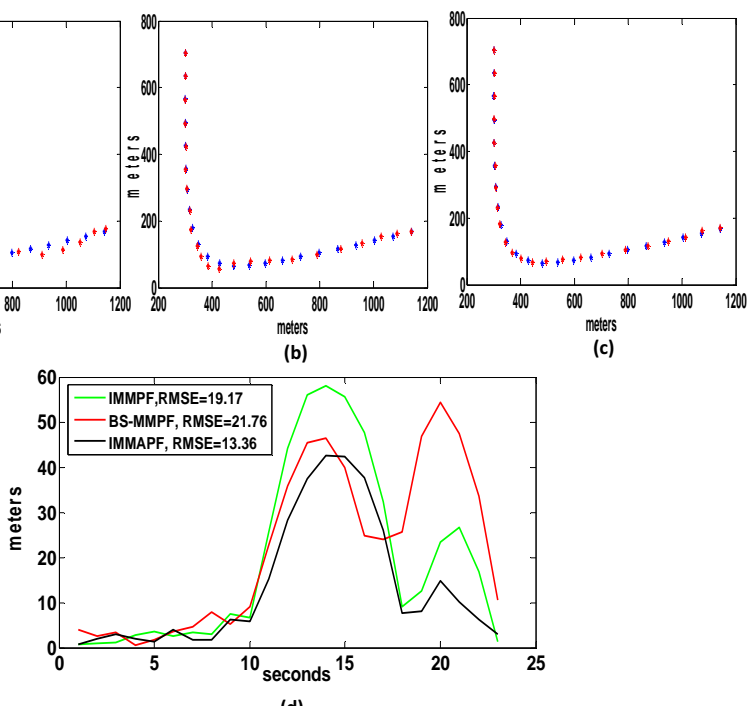

Figure 4. The tracking results of (a).BS-MMPF (b). IMMPF (c). IMMAPF on the ground plane without road information. The blue star represents the vehicle's real positions and the red star represents the tracked ones. (d) represents the corresponding RMSEs for the three algorithms.

Next we compare the three algorithms by incorporating the road information, and the results are presented in Figure 5. We can see that for each algorithm, much lower RMSE is obtained when the road information is applied. And our proposed IMMAPF method, still achieves the best performance among all the three methods after the incorporation of the road information. 


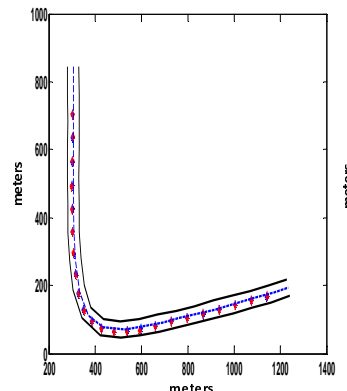

(a)

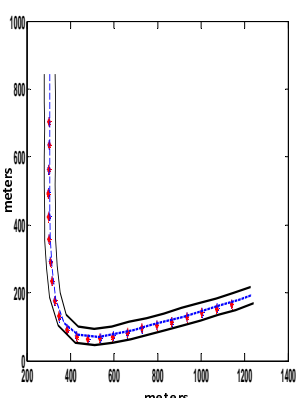

(b)

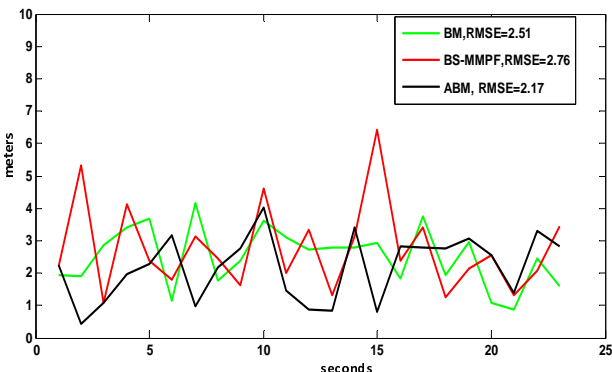

(d)

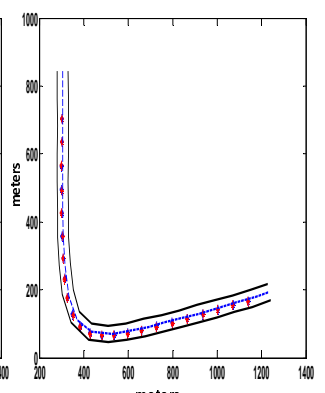

(c)

Figure 5. The tracking results of (a).BS-MMPF (b). IMMPF (c). IMMAPF on the ground plane with road information. The blue star represents the vehicle's real positions and the red star represents the tracked ones. (d) represents the corresponding RMSEs for the three algorithms.

For a more comprehensive evaluation, in each scenario (the road information is applied or is not), 30 Monte-Carlo simulations were performed for each algorithm. The mean and variance of the calculated RMSE are listed in Tables 1 and 2. From these two tables, we can see that much better performance could be achieved by incorporating the road information with much smaller mean and standard derivation of RMSEs for each algorithm. Besides, for the algorithm comparison, our proposed algorithm achieves better performance than the other two algorithms for both scenarios with/without the road information.

Table 1. Performances of different algorithms without road information

\begin{tabular}{|c|c|c|c|}
\hline & BS-MMPF & IMMPF & IMMAPF \\
\hline Mean & 14.68 & 12.88 & 7.40 \\
\hline Standard deviation & 6.95 & 6.68 & 4.55 \\
\hline
\end{tabular}

Table 2. Performances of different algorithms with road information

\begin{tabular}{|c|c|c|c|}
\hline & BS-MMPF & IMMPF & IMMAPF \\
\hline Mean & 2.42 & 2.42 & 2.20 \\
\hline Standard deviation & 0.39 & 0.26 & 0.14 \\
\hline
\end{tabular}




\section{CONCLUSIONS}

In this work, we proposed a novel IMMAPF algorithm for the ground vehicle tracking problem. The auxiliary particle filter was incorporated into the IMM Bayesian filtering framework, which can thereby achieve better performance by taking account the measurement information. The road information was also applied as domain knowledge to constrain the movement of the tracked vehicle for achieving a more accurate tracking result. From multiple Monte-Carlo simulations, it was shown that better ground vehicle tracking performance could be obtained with the aid of the road information. And our proposed algorithm achieved better performance over the traditional ones.

However, we need to consider a more realistic scenario for GMTI tracking. For example, the vehicle will not be detected by the GMTI radar if the measurements are within the "Doppler Blindness Zone" [10]. Besides, some false alarm measurements may occur due to the effect of clutter in the background. After introducing such degraded measurements and false alarms, the measurement model becomes more complicated and one possible solution to deal with it is random finite set theory [12], which takes the measurements as a random finite set.

\section{References}

[1] Mertens, M. and Ulmke, M., "Precision gmti tracking using road constraints with visibility information and a refined sensor model," 2008 IEEE Radar Conference, Rome, Italy (2008).

[2] Ristic, B., Arulampalam, S., and Gordon, N., "Beyond the kalman filter: Particle filters for tracking applications," Norwood, MA: Artech House (2004).

[3] Mazor, E., Averbuch, A., Shalom, Y., and Dayan, J., "Interacting multiple model methods in target tracking: a survey," IEEE Transactions on Aerospace and Electronic Systems 34(1), 103-123 (1998).

[4] Boers, Y. and Driessen, J., "Interacting multiple model particle filter," IEE Proceedings on Radar, Sonar and Navigation 150(5), 344-349 (2003).

[5] Blom, H. and Bloem, E., "Exact Bayesian and particle filtering of stochastic hybrid systems," Aerospace and Electronic Systems, IEEE Transactions on 43(1), 55-70 (2007).

[6] Arulampalam, M., Orton, M., and Ristic, B., "A variable structure multiple model particle filter for GMTI tracking," Proceedings of the Fifth International Conference on Information Fusion, Annapolis, MD, USA (2002).

[7] Ulmke, M. and Koch, W., "Road-map assisted ground moving target tracking," IEEE Transactions on Aerospace and Electronic Systems 42(4), 1264 -1274 (2006).

[8] Cheng, Y. and Singh, T., "Efficient particle filtering for road-constrained target tracking," IEEE Transactions on Aerospace and Electronic Systems 43(4), 1454 -1469 (2007).

[9] Merwade, V., Maidment, D., and Hodges, B., "Geospatial representation of river channels," Journal of Hydrologic Engineering 10(3), 243-251 (2005).

[10] Clark, J., Kountouriotis, P., and Vinter, R., "A new Gaussian mixture algorithm for GMTI tracking under a minimum detectable velocity constraint," IEEE Transactions on Automatic Control 54(12), 2745-2756 (2009). 
[11] Liu, C. and Chen, B. L. W., "Road network based vehicle navigation using an improved IMM particle filter," 2013 IFAC Intelligent Autonomous Vehicles Symposium, Gold Coast, Australia (2013).

[12] Vo, B., Vo, B., and Cantoni, A., "Bayesian filtering with random finite set observations," IEEE Transactions on Signal Processing 56(4), 1313-1326 (2008). 